\title{
Gravitational lensing by spherically symmetric lenses with angular momentum
}

\author{
M. Sereno ${ }^{1}$ and V. F. Cardone ${ }^{2}$ \\ 1 Dipartimento di Scienze Fisiche, Università degli Studi di Napoli "Federico II", and Istituto Nazionale di Fisica Nucleare, \\ Sez. Napoli, via Cinthia, Compl. Univ. Monte S. Angelo, 80126 Napoli, Italia \\ 2 Dipartimento di Fisica "E.R. Caianiello", Università di Salerno, and Istituto Nazionale di Fisica Nucleare, sez. Napoli, \\ gr. Salerno, via S. Allende, 84081 Baronissi (Salerno), Italia
}

Received 31 July 2002 / Accepted 11 August 2002

\begin{abstract}
Following Sereno (2002a), we discuss the bending of light rays by spherically symmetric lenses with angular momentum. For several astrophysical systems, such as white dwarfs and galaxies, gravitomagnetism induces a correction on the deflection angle as large as $0.1 \%$.
\end{abstract}

Key words. astrometry - stars: rotation - cosmology: theory - cosmology: gravitational lensing

\section{Introduction}

Gravitational lensing is one of the best investigated phenomena of gravitation. In the framework of general relativity, its lowest-order predictions have been confirmed by observative astrophysics on very different scales. On the other hand, the impressive development of technical capabilities demands for a full treatment of lensing theory to any order of approximation. The study of higher order perturbative terms is the link between weak and strong regimes of the theory.

Mass-energy currents relative to other masses generate space-time curvature. This phenomenon, known as intrinsic gravitomagnetism, is a new feature of general relativity and other conceivable alternative metric theories of gravity and cannot be deduced by a motion on a static background (for a detailed discussion on gravitomagnetism we refer to Ciufolini $\&$ Wheeler 1995). In particular, the effect of the angular momentum of the deflector has been studied by several authors (Epstein \& Shapiro 1980; Ibáñez \& Martín 1982; Ibáñez 1983; Dymnikova 1986; Glicestein 1999; Sereno 2002a).

One of us (Sereno 2002a) showed how the gravitomagnetic correction to the lensing quantities can be evaluated in the usual framework of lensing theory (see also Capozziello et al. 1999), i.e. $i$ ) weak field and slow motion approximation for the lens; ii) thin lens hypothesis (Schneider et al. 1992; Petters et al. 2001).

In this Paper, we consider the gravitomagnetic contribution to the deflection angle for extended gravitational lenses

Send offprint requests to: M. Sereno, e-mail: sereno@na.infn.it of astrophysical interest. We discuss spherically symmetric deflectors.

\section{The deflection angle}

As derived in Sereno (2002a), the deflection angle, to the or$\operatorname{der} c^{-3}$, is

$\boldsymbol{\alpha}(\boldsymbol{\xi}) \equiv \frac{4 G}{c^{2}} \int_{\mathfrak{R}^{2}} \mathrm{~d}^{2} \xi^{\prime} \Sigma\left(\boldsymbol{\xi}^{\prime}\right)\left(1-2 \frac{\left\langle\boldsymbol{v} \cdot \boldsymbol{e}_{\mathrm{in}}\right\rangle_{l}\left(\boldsymbol{\xi}^{\prime}\right)}{c}\right) \frac{\boldsymbol{\xi}-\boldsymbol{\xi}^{\prime}}{\left|\boldsymbol{\xi}-\boldsymbol{\xi}^{\prime}\right|^{2}} ;$

$\xi$ is the bi-dimensional position vector in the lens plane, orthogonal to the line of sight $l ; \boldsymbol{e}_{\text {in }}$ is the incoming light ray direction; $G$ is the Newton's constant of gravitation; $c$ is the speed of light. $\Sigma$ is the surface mass density projected along the line of sight

$\Sigma(\boldsymbol{\xi}) \equiv \int \rho(\boldsymbol{\xi}, l) \mathrm{d} l$

$\left\langle\boldsymbol{v} \cdot \boldsymbol{e}_{\text {in }}\right\rangle_{l}$ is the weighted average, along the line of sight, of the component of the velocity $v$ of the mass element orthogonal to the lens plane,

$\left\langle\boldsymbol{v} \cdot \boldsymbol{e}_{\text {in }}\right\rangle_{l}(\boldsymbol{\xi}) \equiv \frac{\int\left(\boldsymbol{v}(\boldsymbol{\xi}, l) \cdot \boldsymbol{e}_{\text {in }}\right) \rho(\boldsymbol{\xi}, l) \mathrm{d} l}{\Sigma(\boldsymbol{\xi})}$.

Let us consider a spherically symmetric lens that rotates about an arbitrary axis, $\hat{\eta}$, passing through its center (i.e. a main axis of inertia). To specify the orientation of the rotation axis, we need two Euler's angles: $\alpha$ is the angle between $\hat{\eta}$ and the $\xi_{2}$ axis; $\beta$ is the angle between the line of sight $\hat{l}$ and the line of nodes defined at the intersection of the $l \hat{\xi}_{1}$ plane and the equatorial plane (i.e., the plane orthogonal to the rotation axis and 
containing the lens center). Using the axial symmetry about the rotation axis, we find

$$
\begin{aligned}
\boldsymbol{v} \cdot \boldsymbol{e}_{\text {in }}\left(\xi_{1}, \xi_{2}, l\right) & =-\omega(R)\left[\xi_{1} \cos \alpha+\xi_{2} \sin \alpha \cos \beta\right] \\
& \equiv-\omega_{2}(R) \xi_{1}+\omega_{1}(R) \xi_{2},
\end{aligned}
$$

where $\omega(R)$ is the modulus of the angular velocity at a distance $R \equiv\left(R_{1}^{2}+R_{2}^{2}\right)^{1 / 2}$ from the rotation axis; $\hat{R}_{1}$ (that, given the spherical symmetry of the system, can be taken along the line of nodes) and $\hat{R}_{2}$ are the axes on the equatorial plane; $\omega_{1}$ and $\omega_{2}$ are the components of $\omega$ along, respectively, the $\xi_{1}$ - and the $\xi_{2}$-axes. It is

$R_{1}=l \cos \beta+\xi_{1} \sin \beta$,

$R_{2}=-l \cos \alpha \sin \beta+\xi_{1} \cos \alpha \cos \beta+\xi_{2} \sin \alpha$.

Let us assume a rigid rotation, $\omega(R)=\omega=$ const. We have

$\left\langle\boldsymbol{v} \cdot \boldsymbol{e}_{\text {in }}\right\rangle_{l}=-\omega_{2} \xi_{1}+\omega_{1} \xi_{2}$.

We can, now, evaluate the integral in Eq. (1); it is,

$$
\begin{aligned}
\alpha_{1}(\xi, \theta)= & \frac{4 G}{c^{2}}\left\{\frac{M(\xi)}{\xi} \cos \theta+\frac{I_{N}(\xi)}{\xi^{2}}\left(\frac{\omega_{2}}{c} \cos 2 \theta-\frac{\omega_{1}}{c} \sin 2 \theta\right)\right. \\
& \left.-M(>\xi) \frac{\omega_{2}}{c}\right\} ; \\
\alpha_{2}(\xi, \theta)= & \frac{4 G}{c^{2}}\left\{\frac{M(\xi)}{\xi} \sin \theta+\frac{I_{N}(\xi)}{\xi^{2}}\left(\frac{\omega_{1}}{c} \cos 2 \theta+\frac{\omega_{2}}{c} \sin 2 \theta\right)\right. \\
& \left.+M(>\xi) \frac{\omega_{1}}{c}\right\} .
\end{aligned}
$$

$\xi$ and $\theta$ are polar coordinates in the lens plane; $M(\xi)$ is the mass of the lens within $\xi$,

$M(\xi)=2 \pi \int_{0}^{\xi} \Sigma\left(\xi^{\prime}\right) \xi^{\prime} \mathrm{d} \xi^{\prime} ;$

$M(>\xi)$ is the lens mass outside $\xi, M(>\xi) \equiv M(\infty)-M(\xi)$; and

$I_{N}(\xi)=2 \pi \int_{0}^{\xi} \Sigma\left(\xi^{\prime}\right) \xi^{\prime 3} \mathrm{~d} \xi^{\prime}$

is the momentum of inertia of the mass within $\xi$ about a central axis. $I_{N} \times \omega_{i}$ is the component of the angular momentum along the $\xi_{i}$-axis.

The gravitomagnetic correction consists of the last two terms in Eqs. (8), (9), both proportional to some components of the angular velocity. Spherical symmetry is broken. In the first contribution, the angular momentum appears; the second one is proportional to the mass outside $\xi$ and can be significant for lenses with slowly decreasing mass density.

\section{The homogeneous sphere}

The gravitational phenomena connected to intrinsic gravitomagnetism are generated by mass-energy currents relative to other masses. The simplest lens model, the pointlike Schwarzschild lens, cannot produce such a peculiar effect since the local Lorentz invariance on a static background does not account for the dragging of inertial frames
(Ciufolini \& Wheleer 1995). General relativity is a classicalnonquantized theory where the classical angular momentum of a particle goes to zero as its size goes to zero. To consider the gravitomagnetic field, we need a further step after the point mass as a lens model, the homogeneous sphere. Let us consider a homogeneous sphere of radius $R$ and volume density $\rho_{0}$. It is

$\Sigma(\xi)=2 \rho_{0} \sqrt{R^{2}-\xi^{2}}$, if $\xi \leq R$,

or $\Sigma(\xi)=0$ elsewhere;

$M(\xi)=M_{\mathrm{TOT}}\left[1-\left(1-\left(\frac{\xi}{R}\right)^{2}\right)^{3 / 2}\right]$, if $\xi \leq R$,

or $M(\xi)=M_{\text {TOT }}$ elsewhere, $M_{\mathrm{TOT}}=\frac{4}{3} \pi R^{3} \rho_{0}$;

$I_{N}(\xi)=I_{N}^{\mathrm{TOT}}\left[1-\left(1-\left(\frac{\xi}{R}\right)^{2}\right)^{1 / 2}\left(1+\frac{1}{2}\left(\frac{\xi}{R}\right)^{2}-\frac{3}{2}\left(\frac{\xi}{R}\right)^{4}\right)\right]$,

or $I_{N}(\xi)=I_{N}^{\mathrm{TOT}}$ elsewhere, $I_{N}^{\mathrm{TOT}}=\frac{8}{15} \pi R^{5} \rho_{0}$;

For light rays outside the lens, $\xi>R$, the deflection angle reduces to

$$
\begin{aligned}
\alpha_{1}(\xi, \theta)= & \frac{4 G}{c^{2}}\left\{\frac{M_{\mathrm{TOT}}}{\xi} \cos \theta+\frac{I_{N}^{\mathrm{TOT}}}{\xi^{2}}\right. \\
& \left.\times\left(\frac{\omega_{2}}{c} \cos 2 \theta-\frac{\omega_{1}}{c} \sin 2 \theta\right)\right\}, \\
\alpha_{2}(\xi, \theta)= & \frac{4 G}{c^{2}}\left\{\frac{M_{\mathrm{TOT}}}{\xi} \sin \theta+\frac{I_{N}^{\mathrm{TOT}}}{\xi^{2}}\right. \\
& \left.\times\left(\frac{\omega_{1}}{c} \cos 2 \theta+\frac{\omega_{2}}{c} \sin 2 \theta\right)\right\} .
\end{aligned}
$$

The gravitomagnetic correction is significant if

$\frac{I_{N}^{\mathrm{TOT}}}{M_{\mathrm{TOT}}} \frac{\omega}{c \xi}=\frac{J}{M_{\mathrm{TOT}} c \xi} \gtrsim 10^{-3}$,

where $J \equiv I_{N} \times \omega$ is the angular momentum. To have a nonnegligible gravitomagnetic effect, the angular momentum of the lens has to be non-negligible compared to the angular momentum of a particle of mass $M_{\text {TOT }}$ and velocity $c$ in a circular orbit of radius $\xi$ around the rotation axis.

Let us consider a lens rotating about the $\xi_{2}$-axis $\left(\omega_{1}=0\right.$, $\left.\omega_{2}=\omega\right)$ and a light ray in the equatorial plane, $\theta=0$. The deflection generated by the gravitomagnetic field is

$\alpha_{\mathrm{GRM}}=\frac{4 G}{c^{3}} \frac{J}{\xi^{2}}$.

The sun bends a light ray grazing its limb, $\xi=R_{\odot}$, by 1.75 arcsec. Given its angular momentum, $J_{\odot} \simeq 1.6 \times$ $10^{48} \mathrm{~g} \mathrm{~cm}^{2} \mathrm{~s}^{-1}$ (Allen 1983), the gravitomagnetic correction is $\sim 0.7 \mu \operatorname{arcsec}$ (see also Epstein \& Shapiro 1980).

For an early type star, $J=10^{2} J_{\odot}\left(\frac{M}{M_{\odot}}\right)^{5 / 3}$ (Kraft 1967). For $M=1.4 M_{\odot}, R=1.1 R_{\odot}$ and for a light ray grazing the limb, $\alpha_{\mathrm{GRM}} \simeq 0.1$ milliarcsec, that is, a correction of $\sim 4 \times 10^{-5}$.

The gravitomagnetic field becomes more significant for a fast rotating white dwarf, where $J \sim \sqrt{0.2 G M^{3} R}$ (Padmanabhan 2001). For $M \sim M_{\odot}, R \sim 10^{-2} R_{\odot}, \xi \sim 6 R$, $\alpha_{\mathrm{GRM}} \simeq 0.03 \mathrm{arcsec}$, that is, a correction of $\sim 10^{-3}$. 


\section{The isothermal sphere}

Isothermal spheres (ISs) are widely used in astrophysics to model systems on very different scales, from galaxy haloes to clusters of galaxies; also, IS can be adopted to study microlensing by non-compact invisible objects in the halo (Sazhin et al. 1996).

Let us consider an IS with a finite core radius $\xi_{\mathrm{c}}$. The surface density is

$\Sigma^{I S}(\xi)=\frac{\sigma_{\mathrm{v}}^{2}}{2 G} \frac{1}{\left(\xi^{2}+\xi_{\mathrm{c}}^{2}\right)^{1 / 2}}$,

where $\sigma_{\mathrm{v}}$ is the velocity dispersion. We have,

$$
\begin{aligned}
& M^{I S}(\xi)=\frac{\pi \sigma_{\mathrm{v}}^{2}}{G} \xi_{\mathrm{c}}\left[\left(1+\left(\frac{\xi}{\xi_{\mathrm{c}}}\right)^{2}\right)^{1 / 2}-1\right], \\
& I_{N}^{I S}(\xi)=\frac{\pi \sigma_{\mathrm{v}}^{2}}{3 G} \xi_{\mathrm{c}}^{3}\left[2+\left(\left(\frac{\xi}{\xi_{\mathrm{c}}}\right)^{2}-2\right)\left(1+\left(\frac{\xi}{\xi_{\mathrm{c}}}\right)^{2}\right)^{1 / 2}\right] .
\end{aligned}
$$

When $\xi_{\mathrm{c}}=0$, we have the singular isothermal sphere (SIS). Then,

$M^{\mathrm{SIS}}(\xi)=\frac{\pi \sigma_{\mathrm{v}}^{2}}{G} \xi$

$I_{N}^{\mathrm{SIS}}(\xi)=\frac{\pi \sigma_{\mathrm{v}}^{2}}{3 G} \xi^{3}$.

Since the total mass is divergent, we introduce a cut-off radius $R \gg \xi$. For the SIS, the deflection angle reduces to

$$
\begin{aligned}
\alpha_{1}^{\mathrm{SIS}}(\xi, \theta)= & 4 \pi\left(\frac{\sigma_{\mathrm{v}}}{c}\right)^{2}\left\{\cos \theta+\frac{\omega_{2}}{c}\left[\xi\left(\frac{\cos 2 \theta}{3}+1\right)-R\right]\right. \\
& \left.-\frac{\omega_{1}}{c} \xi \frac{\sin 2 \theta}{3}\right\}, \\
\alpha_{2}^{\mathrm{SIS}}(\xi, \theta)= & 4 \pi\left(\frac{\sigma_{\mathrm{v}}}{c}\right)^{2}\left\{\sin \theta+\frac{\omega_{1}}{c}\left[\xi\left(\frac{\cos 2 \theta}{3}-1\right)+R\right]\right. \\
& \left.+\frac{\omega_{2}}{c} \xi \frac{\sin 2 \theta}{3}\right\} .
\end{aligned}
$$

The correction couples kinematics, through the angular velocity, and geometry, through the cut-off radius. As can be easily seen, the gravitomagnetic effect is significant when

$\frac{\omega}{c} R \gtrsim 10^{-3}$.

In particular, in the inner regions $(\xi \ll R)$, the above equations reduce to

$\alpha_{1}^{\mathrm{SIS}}(\xi \ll R, \theta)=4 \pi\left(\frac{\sigma_{\mathrm{v}}}{c}\right)^{2}\left\{\cos \theta-\frac{R \omega_{2}}{c}\right\}$,

$\alpha_{2}^{\mathrm{SIS}}(\xi \ll R, \theta)=4 \pi\left(\frac{\sigma_{\mathrm{v}}}{c}\right)^{2}\left\{\sin \theta+\frac{R \omega_{1}}{c}\right\} ;$

the correction derives from the mass outside the considered radius.

We can model a typical galaxy as a SIS with $\sigma_{\mathrm{v}} \sim$ $200 \mathrm{~km} \mathrm{~s}^{-1}, R \lesssim 50 \mathrm{kpc}$ and $J \equiv I_{N}(R) \times \omega \sim 0.1 M_{\odot} \mathrm{kpc}^{2} \mathrm{~s}^{-1}$, as derived from numerical simulations (Vitviska et al. 2001). It is,

$\frac{\omega}{c} R \sim \frac{G}{c^{3}} J\left(\frac{c}{\sigma_{\mathrm{v}}}\right)^{2} R^{-2} \sim 10^{-3}$.

The gravitomagnetic correction is quite significant, increases with the ordered motion of the stars (i.e., with the angular momentum) and decreases with the random proper motions (i.e., with the dispersion velocity).

\section{Power law models}

Power law models can be considered as a generalization of the IS (Schneider et al. 1992) and are often adopted to model mass distribution in clusters of galaxies by lensing inversion (Sereno 2002b). It is

$\Sigma^{\mathrm{PL}}=\Sigma_{0} \frac{1+p\left(\frac{\xi}{\xi_{\mathrm{c}}}\right)^{2}}{\left(1+\left(\frac{\xi}{\xi_{\mathrm{c}}}\right)^{2}\right)^{2-p}}$

the slope parameter $p$ determines the softness of the mass profile of the lens. A power law model with $p=1 / 2$ approximates the isothermal sphere at large radius. It is,

$$
\begin{aligned}
M^{\mathrm{PL}}(\xi)= & \pi \Sigma_{0} \xi^{2}\left[1+\left(\frac{\xi}{\xi_{\mathrm{c}}}\right)^{2}\right]^{p-1}, \\
I_{N}^{\mathrm{PL}}(\xi)= & \frac{\pi \Sigma_{0} \xi_{\mathrm{c}}^{4}}{p(1+p)}\left[( 1 + ( \frac { \xi } { \xi _ { \mathrm { c } } } ) ^ { 2 } ) ^ { p - 1 } \left(1+(1-p)\left(\frac{\xi}{\xi_{\mathrm{c}}}\right)^{2}\right.\right. \\
& \left.\left.+p^{2}\left(\frac{\xi}{\xi_{\mathrm{c}}}\right)^{4}\right)-1\right] .
\end{aligned}
$$

\section{Summary and discussion}

We have investigated the effect of dragging of inertial frames in gravitational lensing for spherically symmetric lenses and a general expression for the deflection angle, to the order $c^{-3}$, has been derived. We have explicitly considered isothermal spheres, power law models and the homogeneous sphere. Both for galaxies and white dwarfs, the gravitomagnetic correction can be as large as $0.1 \%$.

The satellite Hipparcos, launched in 1989 by ESA, can measure the position of stars with accuracy of nearly a milliarcsec. New generation space interferometric mission, such as SIM by NASA (scheduled for launch in 2009), should greatly improve this accuracy. Measurements of deflection of electromagnetic waves could give one of the first experimental evidences of gravitomagnetism.

\section{References}

Allen, C. W. 1983, Astrophysical Quantities (The Athlone Press, London)

Capozziello, S., Lambiase, G. Papini, G., \& Scarpetta, G. 1999, Phys. Lett. A, 254, 11 
Ciufolini, I., \& Wheeler, J. A. 1995, Gravitation and Inertia (Princeton University Press, Princeton)

Dymnikova, I. 1986, Relativity in Celestial Mechanics and Astrometry, ed. J. Kovalevsky, \& A. Brumberg, 411

Epstein, R., \& Shapiro, I. 1980, Phys. Rev. D, 22, 2947

Glicenstein, J. F. 1999, A\&A, 343, 1025

Ibáñez, J. 1983, A\&A, 124, 175

Ibáñez, J., \& Martín, J. 1982, Phys. Rev. D, 26, 384

Kraft, R. P. 1967, ApJ, 150, 551

Padmanabhan, T. 2001, Theoretical Astrophysics, vol. II (Cambridge University Press, Cambridge)
Petters, A. O., Levine, H., \& Wambsganss, J. 2001, Singularity Theory and Gravitational Lensing (Birkhäuser, Boston)

Sazhin, M. V., Yagola, A. G., \& Yakubov, A. V. 1995, Phys. Lett. A, 208, 276

Schneider, P. J., Ehlers, J., \& Falco, E. E. 1992, Gravitational Lenses (Springer-Verlag, Berlin)

Sereno, M. 2002a, Phys. Lett. A., in press [astro-ph/0209148]

Sereno, M. 2002b, A\&A, 393, 757

Vitviska, M., Klypin, A., Kravtsov, A. V., et al. 2001, ApJ, submitted [astro-ph/0105349] 